\title{
Sondeo exploratorio sobre el impacto de la incorporación de México al NAFTA en los flujos de nuevas inversiones extranjeras directas hacia Centroamérica
}

\author{
Ernesto Galdámez
}

\section{Anlecedentes}

Las negociaciones para integrar a México en un único megamecardo que cubriría desde el Río Yukon hasla la Peninsula de Yucalán, reuniendo 365 millones de canadienses, mexicanos y estadounidenses en un solo bloque, han cautivado en buena medida la atención de los inversionistas a nivel mundial. En un movimiento anlicipado a la firma del pacto de libre comercio, varias subsidiarias de firmas transnacionales localizadas en Asia han empezado a desmontar sus líneas de ensamblaje para emigrar hacia la nación Azteca. Ejemplos de ello abundan. Se tiene documentado el caso de la compañia American Telephone and Telegraph Co. En vez de expandir su planla en Singapur, ha programado abrir una tábrica de 1500 empleados en Guadalajara a mediados de 1991, para ensamblar máquinas para contestar teléfonos. Del mismo modo, esta compañía está mudando la reparación de teléfonos inalámbricos también de Singapur hacia México, como una estrategia para acelerar los servicios de atención al cliente. Paralelamente Odyssey International Lid., una empresa eslablecida en China por muchos afios para producir una amplia gama de prendas de vestir y de arliculos deportivos destinados a suplir el mercado norteamericano, está 
transfiriendo varias de sus operaciones hacia México. En principio, tiene planes de abrir alli tres nuevas plantas para tines de este año. De manera similar Inter-American Holdings Co. y Proxima Corp., eslán Irasladando desde China y Taiwan respectivamente, muchas de sus líneas de producción hacia México.

Ya ubicados en ese contexto, el 12 de agosto del presente año la sefiora embajadora Carla Hills, representante comercial de los Eslados Unidos de América, se reunió en la ciudad de San José, Costa Rica, con representantes de los sectores privados y públicos centroamericanos para disculir ampliamenle con ellos varios aspectos estratégicos de la Iniciativa para las Américas, lanzada por el Presidente Bush, en junio de 1990. Dentro de los muchos puntos de agenda abordados durante la reunión, FEDEPRICAP puso sobre la mesa de discusión los temores abrigados por todo el sector privado del istmo en relación a las negociaciones que se están realizando para la incorporación de México al Area Norleamericana de Libre Comercio (NAFTA). Se planleó que se veian "esas negociaciones como un paso muy positivo en la liberalización del comercio, un paso de proporciones históricas y enorme ińluencia positiva a largo plazo. Pero no podemos evitar preocuparnos sobre los prospectos que encararemos si los benelicios que México recibirá al incorporarse al Area Norteamericana de Libre comercio, no son compensados de alguna lorma, o compensados en forma simultánea, a los países de la Cuenca del Caribe. Los efectos podrían ser devastadores y probablemente podrian poner en peligro lodos los logros que la Iniciativa de la Cuenca del Caribe ha generado. De hecho, ya hay inversionistas polenciales extranjeros que eslán revisando sus planes de inversión, lo que es evidente en el sector maquila que desde ya, está sintiendo esos efectos debido a que varios inversionistas potenciales, incluyendo de Eslados Unidos de América y Asia, han cancelado sus estudios de inversión". Los ejemplos citados en el párralo inicial atizan tales temores y son signos no descartables de la ocurrencia de un tenómeno de desviación de inversiones.

Básicamente, la posición oficial asumida por la oficina del represenlate comercial de los Estados Unidos Irenle a tales planteamienlos, ha sido la siguiente. Dado que los términos especílicos a ser alcanzados por los grupos de trabajo en las seis áreas de negociación todavia estan por definirse, muchas de las afirmaciones sostenidas sobre las consecuencias a derivarse de ellos están fincadas en el lerreno especulativo. También disculen que aún no eslá lo suficientemente claro si el NAFTA causará alguna dislocación de corto plazo sobre los flujos de inversión hacia la región. No creen que los países de la Cuenca del Caribe saldrán perjudicados ostensiblemente en su capacidad de atraer nuevas 
companias internacionales, a raíz de la supuesla erosión de las ventajas compelitivas de sus exportaciones, primordialmenle no tradicionales, por los beneficios prelerenciales a concederse a México bajo el NAFTA.

FEDEPRICAP, considerando la envergadura de las posibles repercuciones negalivas que podría ocasionar un fenómeno de tal naturaleza sobre los niveles de actividad económica en las sociedades centroamericanas, estimó de suma urgencia proceder a la larea de realizar un estudio regional cuyo objetivo luese el de comprobar empiricamente la existencia de esas desviaciones de inversión en cuestión.

Este breve trabajo busca recabar evidencia empirica sobre casos de empresas extranjeras que hayan decidido suspender temporalmente o hayan abandonado por complelo sus proyectos de invertir en Centroamérica para iniciar operaciones destinadas a exportar hacia lerceros mercados, alraídos especialmenle por las nuevas oportunidades de negocios abiertos México con su probable incorporación al gran bloque comercial de América del Norte. De esta manera se pretende descubrir en la práclica si han ocurrido desviaciones en los flujos de inversión extranjera originalmente planeados a realizarse en Centroamérica, redeslinados hacia México con motivo de su esperada asociación al NAFTA.

\section{Hallazgos prellminares}

\subsection{Costa Rlca}

La temática tocada por la investigación les pareció algo tolalmente ajeno a su quehacer institucional. De acuerdo a su sislema administrativo de seguimienlo, del conjunto de empresas extranjeras traidas al país durante el último ano por su programa de promoción de inversiones para sondear las posibilidades de inslalar alguna línea de producción, ninguna ha sido reportada por haber desistido en su inlerés de invertir localmente para desplazar su proyecto hacia México.

En sus archivos, no se registra ningún caso concrelo que pudiera ser abonado como constatación positiva de la existencia de tales desviaciones de inversión. A decir verdad, genieralmente aquellas empresas que visitaron el pais y decidieron no inslalar aqui sus plantas, se han mostrado muy reservadas en cuanlo a revelar su opción prelerencial por invertir en olro lugar.

El tipo de información recopilada contrastó marcadamente con la esperada. A priori, se creía que por ser Costa Rica el polo de Centroamérica donde la alluencia de capilal es mayor debido en gran medida a 
su bajo riesgo político, y por ser CINDE la instilución privada de más trayectoria y experiencia en la alracción de empresas extranjeras, era lógico suponer una más alla probabilidad de enconlrar allí una valiosa luente de datos para eliminar la hipótesis de Irabajo manejado en esta investigación. Sin lugar a dudas la evidencia enconlrada en CINDE ayudó enormemente a su comprobación efectiva, pero en el sentido de socavarle lundamenlos a su validez.

\subsection{Honduras}

Los resultados arrojados por las indagaciones hechas en San Pedro Sula, mostraron un cambio de fortuna. En los registros de la oficina de FIDE allí ubicada, se lograron idenlificar con "nombre y apellido" (ver cuadro No. 1) los casos de dos empresas, curiosamente ambas ubicadas en San Juan de Puerlo Rico para atender el mercado del Caribe, las cuales duranle su visita exploraloria a Honduras declararon muy explícilamente su inclinación a irse mejor a instalar sus operaciones en México. Este fue el caso de Stanric, Inc. y G.H. Bass.

A un nivel ya de detalle se supo que por ejemplo, la Empresa Slanric, Inc., filial de Standard Motor Producls, Inc., dedicada a la manufaclura de piezas mecánicas para el sistema de carburación de motores para aulomóviles adujo con mucha Iranqueza no únicamente la posibilidad de libre acceso al mercado norleamericano o los coslos más bajo de la mano de obra vis-a-vis los Estados Unidos como determinantes para escoger a México como silio para su inversión. También trajo a colación la importancia, capilal para ellos, de contar con las economias externas ofrecidas por toda una instraestructura industrial que les provee de insumos directos a su proceso productivo. Esta última no existia en Honduras.

No obstante haberse delectado la evidencia de la fuga de las dos empresas mencionadas, lo cual podría dar pie a un fundado temor de estar en presencia de la punta de iceberg de un fenómeno de grandes proporciones, los ejeulivos enlrevislados de FIDE no mosiraron signos de encontrarse alarmados por al eventual incoporación de México al NAFTA y sus secuelas negativas sobre los flujos de inversión extranjera hacia la región centroamericana. Cuantilativamente hablando, hoy por hoy tal luga signilicó para Honduras la no creación de 250 nuevas plazas de trabajo para su población.

Mucha de la confianza institucional de FIDE en no salir muy perjudicados por esla nueva configuración de la competencia por atraer hombres de negocios para abrir planlas de producción orientada hacia la exportación, radica principalmente en la consideración de tener bien di- 


\section{Cuadro 1}

Casos de empresas que reaizaron una vislla exploratoria a Centroamérica pero decidieron invertir en México

\begin{tabular}{|c|c|c|c|}
\hline Concepto & \multicolumn{3}{|c|}{ Empresas Identiflcadas } \\
\hline Pazón Social & G.H. Bass Caribeean Inc. & Stanfic. Inc. & $\begin{array}{l}\text { Sara Lee } \\
\text { Knit Products. }\end{array}$ \\
\hline Dirección & $\begin{array}{l}\text { Poad No. 2, Kn. 49, } 2 \\
\text { P.O. Box } 365 \\
\text { Manatí, Puerto Rico } \\
00701\end{array}$ & $\begin{array}{l}\text { Call Box } 128 \\
\text { Puerto Real Station } \\
\text { Fajardo, Puerto } \\
\text { rico 00740-0128 }\end{array}$ & \\
\hline Fax y Tel. & $\begin{array}{l}\text { Fax.: (809) } \\
854-3040\end{array}$ & $\begin{array}{l}\text { Fax.: }(809) \\
863-8130\end{array}$ & $\begin{array}{l}\text { Tel.: (919) 744-7893 } \\
\text { Fax.: }(919) 744-3471\end{array}$ \\
\hline $\begin{array}{l}\text { Represen- } \\
\text { tante }\end{array}$ & $\begin{array}{l}\text { Luis Benitez, Jr. } \\
\text { Vice-President and } \\
\text { General Manager }\end{array}$ & $\begin{array}{l}\text { Louis Tebodo } \\
\text { General Manager }\end{array}$ & Julio A. Barea \\
\hline Pals visitado & Honduras & Honduras & El Salvador \\
\hline $\begin{array}{l}\text { Compañla } \\
\text { matriz }\end{array}$ & $\begin{array}{l}\text { Van Heusen } \\
\text { Productora de } \\
\text { zapatos BASS }\end{array}$ & $\begin{array}{l}\text { Standard Motor } \\
\text { Produets. Ine. } \\
\text { Productora de } \\
\text { partes de carros }\end{array}$ & Sara Lee \\
\hline $\begin{array}{l}\text { Actividad } \\
\text { proyectada a } \\
\text { realizar }\end{array}$ & $\begin{array}{l}\text { Ensamblado de } \\
\text { zapatos }\end{array}$ & $\begin{array}{l}\text { Ensamblaje de } \\
\text { partes del sistema } \\
\text { de inyeccion para } \\
\text { carburadores }\end{array}$ & $\begin{array}{l}\text { Maquila de ropa } \\
\text { y conlección }\end{array}$ \\
\hline $\begin{array}{l}\text { Estimado de } \\
\text { No. de } \\
\text { empleos } \\
\text { realizar }\end{array}$ & 200 & 150 & 100 \\
\hline Statuts & $\begin{array}{l}\text { Esta cvompañla añadio } \\
\text { a México en sus evalua- } \\
\text { ciones pero todavla con- } \\
\text { sidera fuertemente a } \\
\text { Centroamérica como } \\
\text { opción para invertir. }\end{array}$ & $\begin{array}{l}\text { Se estableció en } \\
\text { México }\end{array}$ & $\begin{array}{l}\text { Esta pendiente su } \\
\text { decisión dependien- } \\
\text { do de cómo resulten } \\
\text { las negociaciones . } \\
\text { NAFTA. }\end{array}$ \\
\hline
\end{tabular}

sefiada su estrategia de promoción internacional. Partiendo del hecho de gozar internamente de un clima político relalivamente estable y pacifico, de disponer de abundante mano de obra con niveles salariales competitivos, de tener un régimen de incentivos fiscales de exoneración 
perpetua del pago de impueslos sobre la renta a los inversionislas extranjeros, de proveer un servicio confiable de electricidad, agua y comunicaciones, de no lener Honduras asignadas cuolas de importación por parte de los Estados Unidos, elc., se han lanzado a contactar y atraer empresas extranjeras dedicadas a la confección de prendas de vestir para atender principalmente el mercado norteamericano.

De acuerdo con sus planes de trabajo, las cifras proyectadas se han venido cumpliendo en la práctica. No han percibido un cambio apreciable en el número de inversionistas atendidos. Las companías asiáticas y norteamericanas han conlinuado arribando al pais para establecerse en las zonas francas $o$ en los Parques Industriales. El boom en el rubro de confección y maquilado de ropa para la exportación ha proseguido su ritmo de crecimienlo. Como muestra de esa presión ejercida por la demanda extranjera por techo industrial, se puede ver la respuesla de la oferta nacional con la puesta en marcha y desarrollo simultáneo de seis nuevas zonas de procesamiento para la exportación, a iniciativa y con recursos provenientes de la empresa privada.

Buena parte del optimismo proviene de sus comunicaciones sostenidas permanentemente con Miguel Rothbaum Presidente de American Apparel Manulacturing of America. El de acuerdo a la informacion circulante en los corrillos de Washington, D.C., eslá convencido sobre la inminente exclusión de los lextiles y confección de ropa del tralado a ser suscsrito en México y los Eslados Unidos. En el peor de los casos, si lales rubros son incluidos éstos gozarán de similares condiciones de tralamiento a las contenidas en el CBI. Además, tomando como punto de referencia la letra menuda del tralado firmado por Estados Unidos y Cánada para la creación de una zona de Libre Comercio se descubre en éste una serie de provisiones de salvaguardia que postergan en el tiempo la apertura inmediata de las fronteras al flujo de productos tales como textiles, puercos y troncos de árboles. Cualquier acuerdo con México es dable esperar, de seguro contendrá similares claúsulas de aplicación.

Están muy conscienles de que México es y será un competidor más fuerte en la arena de la atración de capitales extranjeros, principalmente por la relorma estructural aplicada a su aparato productivo y por las ventajas ofrecidas por su proceso de insersión agresiva en el sislema global de intercambio a escala mundial. Esio será bastante evidenle en aquellas áreas donde México ha logrado acumular varias ventajas comperativas con la sofistificación de muchas de las actividades industriales desarrolladas bajo su modelo de producción compartida inslalada a lo largo de la gran zona maquiladora de su frontera norte. No cabe ninguna duda, además, sobre la puesla en una posición competitiva 
desventajosa a muchos paises del $\mathrm{CBI}$ por la concesión de beneficios arancelarios y no arancelarios para muchos artículos producidos en México por su adhesión al NAFTA.

A efectos de proseguir excarvando un poco más hondo dentro de su sistema inslitucional de informática para descubrir si existia una mayor cantidad de datos sobre el tema en cuestión FIDE se compromelió a continuar sobre la pista del problema. Se pusieron de inmediato en conlacto con las oficinas de su departamento de promoción externa localizadas en las ciudades de Allanta, Miami y Nueva York. Se requerió de su ayuda para ver si era faclible que ellos suministraran un listado de empresas cuyos casos particulares trajeran más evidencia empírica a la presente investigación. Dicha solicilud no los agarró por sorpresa, ni tampoco les resultó algo tolalmente extrano. Reporlaron haber desarrollado su trabajo de promoción con al menos 5 companías (ver cuadro 2) interesadas en principio en explorar seriamenle la posibilidad de establecer en Honduras algún lipo de manufacluras, las cuales habian decidido al final del proceso escoger a México como un lugar más propicio para los intereses de la firma, anlicipando su adherencia al NAFTA.

A primera vista esle hallazgo pudiera tomarse como la prueba testimonial, irrefutable, de la validez de las preocupaciones compartidas tanto por los gobiernos como por los sectores privados centroamericanos. Ahora se podria alegar que supuestos efectos negativos colaterales esperados en la región por la recanalización hacia México de corrientes importantes de inversión extranjera direcla, han abandonado la eslera de la mera dimensión psicológica para devenir en realidades consumadas. Hoy ya no se estaría hablando de meras presunciones fundadas en probabilidades, sino de lidiar con la ocurrencia innegable de un fenómeno cuyas magnitudes y consecuencias económicas son verdaderamente desconocidas.

No obstanle lo anterior, al examinar más Iriamente la información recopilada resaltan varias características a comentar. En primer lugar, no se sabe con certeza cuáles fueron las razones específicas para que las empresas prefirieran abandonar sus intenciones de venir a Honduras. Unicamenle se sabe que Fruit of the Loom y Oneita Industries se establecieron en México. Punto. Por su lado, Wilkings Industries da la impresión de haber andado buscando otro tipo de arreglo para su negocio. Lo cierto es que se involucró en una coinversión en México. En cualquier caso, para las tres empresas releridas se necesitaria un mayor estudio para saber cómo y si acaso el factor NAFTA intervino en sus decisiones. En segundo lugar, el lipo y el contenido mismo de la información proveida por las oficinas de FIDE en el exterior no es lo suficien- 
Cuadro 2

Empresas contactadas en el exterlor que preflrieron desvlar su atenclón de Honduras hacla Méxlco por la posibilldad de su Incorporación al NAFTA.

\begin{tabular}{|c|c|c|c|c|c|}
\hline \multirow{2}{*}{$\begin{array}{l}\text { Concepto } \\
\text { Razón Social }\end{array}$} & \multicolumn{5}{|c|}{ Contenldo } \\
\hline & Fuir of the Loom & Wilkings Industries & Oneila Industries & IMAT & Lucky Slar \\
\hline Dirección & $\begin{array}{l}\text { Fuir of hte Loom Drive } \\
\text { Howling Green KY } \\
42103\end{array}$ & $\begin{array}{l}\text { P.O. Box } 1512 \text { Athens, } \\
\text { Georgia } 30603\end{array}$ & $\begin{array}{l}\text { P.O. box } 24 \text { Andrewn. } \\
\text { S. C. } 29510\end{array}$ & $\begin{array}{l}15050 \text { Delano St. } \\
\text { Los Angeles, Calil. } \\
91411\end{array}$ & $\begin{array}{l}311 \text { Est De Leon St. } \\
\text { Tampa, Florida } \\
33609\end{array}$ \\
\hline Telélono & (502) $781-6400$ & (404) 646-7960 & (803) $264-5225$ & (818) 901-1416 & (813) $877-1133$ \\
\hline $\begin{array}{l}\text { Nombre del } \\
\text { conlacto }\end{array}$ & Alake Tarpley & William Gilmore & Larry Lambert & Harry Haralambus & Perry Keene \\
\hline $\begin{array}{l}\text { Lineas de } \\
\text { produclos }\end{array}$ & Prendas de vestir & Prendas de vestir & Prendas de vestir & Prendas de vestir & Prendas de vestir \\
\hline Situación & $\begin{array}{l}\text { Establecida en } \\
\text { México }\end{array}$ & $\begin{array}{l}\text { Hizo una coinversión } \\
\text { en México }\end{array}$ & $\begin{array}{l}\text { Tomó la decisión de } \\
\text { establecerse en } \\
\text { México }\end{array}$ & $\begin{array}{l}\text { Esta compañia re- } \\
\text { presenta clientes } \\
\text { asiáticos. Ellos iban } \\
\text { a viajar a Honduras } \\
\text { en abril. En vez de } \\
\text { ello viajaron a } \\
\text { México y establecie- } \\
\text { ron alli una planta } \\
\text { manulacturera. }\end{array}$ & $\begin{array}{l}\text { La decisión de esta } \\
\text { compañia está en } \\
\text { suspenso debido al } \\
\text { NAFTA de México. }\end{array}$ \\
\hline
\end{tabular}


temente amplio y confiable como para extraer sólidas conclusiones. Tenemos el caso de G.H. Bass (ver cuadro 1). Esta empresa inicialmente se reportó haber desertado de su proyecto de eslablecerse en Honduras. Pero según información de última hora, G.H. Bass sólo añadio a México enlre sus alternativas de inversión pero siguen fuertemente considerando venir a Centroamérica. Tomemos otro ejemplo de la ambigüedad conlenida en la información manejada. La empresa IMAT es representante de varios clientes asiáticos. Ellos tenian planeado visitar Honduras en abril. En vez de eso viajaron a México y establecieron allí una planta manulacturera. Sin embargo, lodavia siguían interesados en visitar Honduras durante el último trimestre de 1991.

\subsection{El Salvador}

Este país no se ha caracterizado precisamente por recibir una afluencia masiva de inversionistas extranjeros durante la última década. EI grado de riesgo e incertidumbre olrecida por las condiciones políticas prevalecienles para la recuperación del capital han disuadido y ahuyenlado en lorma sistemática el arribo de un número significativo de empresas inlemacionales. No obstanle, algunas de ellas individualmente han venido a sondear las oportunidades de negocio; olras se han aventurado a abrir allí sus operaciones.

En un contexto de similar naturaleza al descrito, se vuelve muy difícil la tarea el querer evaluar con toda objetividad el impacto de la polencial incorporación de México al NAFTA sobre los flujos de inversión externa hacia El Salvador, cuando en realidad el comportamiento de esta variable se encuentra sobredelerminado por la presencia de factores extraeconómicos. Por suerte, para los fines de la presente investigación se logró recopilar el caso de la compania Sara Lee Knit Products (ver cuadro 1). Esla es una empresa dedicada a la confección de prendas de vestir cuyos representantes habian manifestado un genuino interés en inaugurar una planta en Centroamérica. El Salvador era su prospecio más atraclivo. Su iniciativa se vio coarlada por el avance de las pláticas bilaterales enlabladas entre México y los Eslados Unidos de América para integrar al primero en el mercado abierto más grande del mundo. En tanlo no se hagan públicos los compromisos finales acordados por ambos gobiernos y se desconozca como se implementará el programa de levanlamiento de los obstáculos al libre flujo de mercancías entre las naciones firmantes, consideran que es poco prudenle ponerse a especular precipilando una decisión. Lo razonable es hacer un compás de espera mientras se vislumbra un panorama más detinido. Por de pronto han optado por celebrar contralos de producción con empresas locales para el maquilado de sus productos. 
El punto medular alrededor del cual gravitan las dudas y se ciernen las expectalivas, es el de saber a ciencia cierla cómo y en qué medida se verá erosionada la competitividad de la oferta de las exportaciones cenlroamericanas en el mercado norteamericano vis-a-vis las mercancías producidas en México, una vez el Tratado Comercial de NAFTA entre en vigencia. En otras palabras, la preocupación se genera por la falla de sulicientes elementos de juicio de carácter objetivo que permitan responder adecuadamente a la preguunta de cómo se verán afeclados los beneficios y concesiones especiales de acceso olorgadas a los produclos de aquellos países beneficiarios de la Iniciativa de la Cuenca del Caribe. Esta incertidumbre provoca el electo de inhibir nuevas inversiones extranjeras cuyo objetivo sea producir localmente para exportar venlajosamente hacia los Estados Unidos. Aún más, el panorama se lorna más lúgubre si adicionalmente tenemos en cuenla que el NAFTA, a diferencia de la Iniciativa de la Cuenca del Caribe, será un acuerdo contractual, con derechos y obligaciones iguales para las partes, y no un pliego de concesiones comerciales unilaterales sin tralamiento recíproco. Esto le agrega una buena dosis de inestabilidad relaliva al gozo de tales benelicios; en cualquier momento éstos podrian ser retirados lotal o parcialmente por el pais olorganle. Como es natural, este componente descrilo obliga al inversionista a reevaluar el horizonte de tiempo en el cual el proyecto podría llegar a ser rentable. A mayor periodo de maduración se requerirá aumentar la lasa de descuento esperada para compensar de ese modo el aumento en el riesgo corrido. Es muy probable que varios proyectos evaluados con este factor incluido, sean desechados por causa de irrentabilidad linanciera.

\subsection{Guatemala}

En las oficinas del centro de Servicios al Inversionista no lue posible recabar evidencia empírica para demostrar el daño causado en el corto plazo por el NAFTA a la recepción en Guatemala de inversión extranjera direcla. De acuerdo a las personas entrevistadas, hasta la lecha no pudieron decir con loda propiedad haber conlactado alguna empresa 0 atendido a alguna persona quien haya manifestado de manera explícita su intención de ya no venir a inslalar aqui su planta procesadora para la exportación, aduciendo, como si let motiv de su resolución, la conveniencia de hacerlo preferiblemenle en México para penetrar más fácitmente el mercado norteamericano al amparo de los polenciales beneficios ofrecidos por el NAFTA. Generalmente, cuando las empresas renuncian a seguir adelante, investigando las posibilidades de invertir, no dan mayores explicaciones o no exponen abiertamente las razones juslificantes de su decisión. 
Ahora bien, no obstanle carecer de datos concretos como los buscados, las personas entrevistadas quisieron dejar constancia de una observación. Quizá por pura coincidencia, o tal vez atribuible a otros faciores, a partir de la firma por los Presidentes Bush y Salinas de Borlari del tralado marco para la creación de una zona de libre comercio, en junio de 1990, han nolado una mengua del interés de los inversionistas exIranjeros en el país. Esto se ha podido apreciar en la disminución del número de ellos que han proseguido pidiendo dalos más puntuales y específicos una vez se les ha proporcionado toda la inlormación general sobre las condiciones de la economía en su conjunlo.

\section{Consideración flnal}

A esta altura del reporle, vale la pena introducir una consideración de mucho peso. Es peligroso querer centrarse a analizar las desviaciones de inversión extranjera de Centroamérica hacia México, tomando la perspectiva de hacer depender en exclusiva a esta úllima variable de la determinante llamada NAFTA. Con centeza este modo de ver las cosas conducirán inexorablemenle a sacar talsas apreciaciones. No se puede abstraer del análisis los programas de ajuste estructural, modernización productiva e inserción en la economía mundial, iniciados en México a principios de los años ochenla. Este proceso de cambio ha sido acelerado y prolundizado con la ascención de Salinas de Gortari a la presidencia. Su proyecto se basa en la premisa de dejar al libre mercado la tarea de organizar el funcionamienlo del sistema económico. Para ello, ha emprendido agresivamente el desmanlelaminto de las murallas proteccionistas, reduciendo las tarilas de imporlación a un promedio del $4 \%$; ha procedido a privatizar masivamente 750 de 1155 empresas estalales; ha rebajado sustancialmente las reglas para el tratamiento a la inversión extranjera; ha recortado enormemente el gasto público y la participación del Estado en economía.

Todo ese plan de reorientación económica de liberalización y volcamiento hacia el exterior, referido eufemísticamenle por muchos como "Salinastroika", ha signilicado la proyección de una lotal nueva imagen de México ante la comunidad internacional de hombres de negocio. El mensaje es claro: México le da la bienvenida a la inversión extranjera, ofreciéndoles una serie de ventajas comparativas. Ya no solo se ofrece la cercania geográfica y un régimen especial de producción compartida con los Estados Unidos como incentivos para su atracción, sino lambién un marco regulatorio institucional diseffado para lal fin. Los resultados obtenidos hablan por si mismos. En la actualidad exislen aproximadamente 2000 maquiladoras instaladas en México, empleando 400,000 personas. 
El propósito mantenido en mente al hacer la anterior acotación es enlatizar, con toda honestidad, los vacios de información encaradas por el presente sondeo exploratorio. Ateniéndono a la calidad de dalos obtenidos durante la investigación, pero sobrelodo apegándonos rigurosamente al análisis objetivo de los hechos, no podriamos incluir inequivocamente estar en el umbral de una declinación sustancial del arribo de capitales exiranjeros frescos a la región cenlroamericana causado por su desviación hacia México. Los dalos disponibles no permiten distinguir si efeclivamente México se ha convertido en una alternativa de inversión que compite direclamente con Centroamérica a raiz de su eventual incorporación al NAFTA, o si acaso esa competencia se ha venido gestando por otro tipo de causas determinantes tales como la implementación de su relalivamente grande y compleja de producción industrial, la cercanía geogrática con los Estados Unidos, o cualquier otro factor percibido como ventaja comparaliva por el inversionista. 